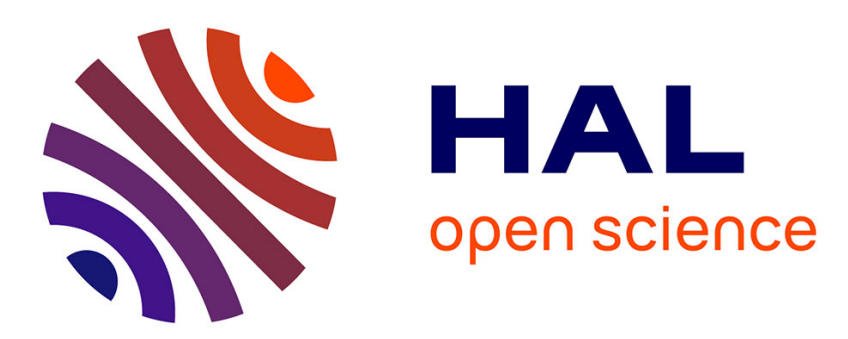

\title{
Des revenus nationaux pour l'Afrique? La mesure du développement en Afrique occidentale française dans les années 1950
}

Vincent Bonnecase

\section{- To cite this version:}

Vincent Bonnecase. Des revenus nationaux pour l'Afrique? La mesure du développement en Afrique occidentale française dans les années 1950. Revue canadienne d'études du développement / Canadian Journal of Development Studies, 2014. hal-01648099

\section{HAL Id: hal-01648099 \\ https://hal.science/hal-01648099}

Submitted on 24 Nov 2017

HAL is a multi-disciplinary open access archive for the deposit and dissemination of scientific research documents, whether they are published or not. The documents may come from teaching and research institutions in France or abroad, or from public or private research centers.
L'archive ouverte pluridisciplinaire HAL, est destinée au dépôt et à la diffusion de documents scientifiques de niveau recherche, publiés ou non, émanant des établissements d'enseignement et de recherche français ou étrangers, des laboratoires publics ou privés. 


\section{Des revenus nationaux pour l'Afrique?}

\section{La mesure du développement en Afrique occidentale française dans les années 1950}

Vincent Bonnecase (CNRS, LAM, Bordeaux, France)

Vincent Bonnecase (2014), « Des revenus nationaux pour l'Afrique ? La mesure du développement en Afrique occidentale française dans les années 1950 ", Canadian Journal of Development Studies / Revue canadienne d'études du développement, 35:1, p. 28-43 
RÉSUMÉ

Cet article interroge les modalités concrètes de production des premiers revenus nationaux en Afrique occidentale française dans les années 1950, leur signification pour les contemporains et les usages antagoniques auxquels ils ont donné lieu. II montre que ces chiffres, hautement incertains au regard de leurs conditions de calcul et du point de vue de leurs propres concepteurs, n'en ont pas moins été rapidement utilisés par différents acteurs, à des fins d'évaluation comptable des politiques coloniales ou de comparaison internationale des niveaux de développement.

\section{ABSTRACT}

This article investigates the methods used for producing the first national revenues in French West Africa during the 1950s, their significance today and the controversial usages which they have led to. The article demonstrates that these figures, highly uncertain in terms of how they were calculated and conceived, were nevertheless rapidly used by different actors to different ends, be it for colonial policy or for comparing international levels of development. 


\section{Introduction}

C'est sans doute une étrange entreprise que de vouloir, en l'an de grâce 1953 et dans un pays de l'Union française où les services officiels de statistique ne sont même pas implantés aux échelons territoriaux, s'attaquer au problème qui figure parmi les plus ardus des économistes modernes : le calcul du revenu national. (Direction générale des Finances de l'AOF, « Essai de récapitulation des éléments connus à Dakar pour servir à un calcul du revenu national de I'AOF », Dakar, 1953)

En 1953, la Direction générale des Finances de l'Afrique occidentale française (AOF)1 entreprend, pour la première fois dans I'histoire de la fédération, de procéder au calcul du revenu national de ce territoire de près de $500000 \mathrm{~km}^{2}$ et d'environ 16 millions d'habitants. Cette initiative, "étrange " au regard de ses propres initiateurs, s'inscrit dans un triple contexte à la fois politique, idéologique et économique. Tout d'abord, à l'échelle de l'AOF ellemême, les autorités coloniales se sont lancées depuis 1946 dans une nouvelle politique d'investissement et de planification qui réforme totalement les relations financières entre la métropole et les colonies : alors que celles-ci avaient jusqu'alors, en vertu d'un principe d'autonomie définie par la loi des finances de 1900, largement autofinancé par le biais de la fiscalité locale leur propre équipement, ce dernier repose désormais également sur les fonds métropolitains (Coquery-Vidrovitch 1982). Ensuite, à l'échelle de la métropole, l'après-guerre voit s'accroître le rôle régulateur de l'État dans les politiques économiques, lesquelles se retrouvent dotées d'un nouveau langage : la comptabilité nationale. Cette évolution induit une valorisation des grands agrégats, au premier rang duquel le produit intérieur brut, désormais établi de manière routinière à des fins de prévision économique globale et de fixation des politiques publiques (Desrosières 2003a). Enfin, à l'échelle internationale, l'émergence d'une idéologie du développement (Rist 1996) va de pair avec une nouvelle demande pour des indicateurs permettant la mesurabilité et la comparabilité des conditions d'existence dans le monde : les espaces coloniaux en particulier se retrouvent inscrit dans une hiérarchisation internationale des niveaux de vie dont ils étaient, jusqu'alors, globalement exclus (Bonnecase 2011).

L'objectif de cet article est de proposer une contribution à l'histoire des indicateurs productifs et à leur place dans la mesure du développement, en revenant sur l'élaboration des premiers revenus nationaux en AOF dans les années 1950, au moment de ce qu'on a pu appeler le colonialisme tardif. II ne s'agira pas, dans une optique rétrospective, de mettre en question ces indicateurs à la lumière de leurs critiques actuelles (Destremeau et Salama 2002 ; Bourmaud 2011), mais de les replacer dans les controverses de l'époque en interrogeant à la fois leurs conditions concrètes d'élaboration, le sens qui a pu leur être donné par les 
contemporains et les premiers usages politiques qui ont été les leurs. Je m’appuierai pour cela, outre la littérature s'inscrivant dans une "sociologie historique de la quantification » (Desrosières 2008a), sur trois principaux types de sources : d'abord, les premières études du revenu national effectuées en AOF, et plus largement en Afrique française, sous l'égide du gouvernement général de la fédération et du ministère de la France d'outre-mer; ensuite et en amont, les données sollicitées dans ces études et préalablement collectées dans le cadre des pratiques routinières de l'administration; enfin et en aval, les discours politiques à plus large diffusion dans lesquels les revenus nationaux ont figuré dès les années 1950, peu après leur élaboration.

Trois questions traverseront cet article. La première porte basiquement sur la propension des premiers revenus nationaux à décrire la réalité économique de l'AOF. On peut vite être amené à affirmer, en s'appuyant sur les doutes formulés à cet égard par les enquêteurs (doutes que l'on peut en même temps facilement retrouver dans n'importe quelle enquête de ce type) que ces chiffres ne veulent pas dire grand-chose au regard de leurs modalités d'élaboration. Mais il ne me semble pas moins important de prendre au sérieux, au moins au départ, la prétention descriptive des premiers concepteurs du revenu national et les débats méthodologiques suscités par cette prétention. La deuxième question porte sur le caractère spécifique qu'a pu revêtir le revenu national dans un contexte colonial et développementaliste, alors que les gouvernements des pays industrialisés procédaient également, à l'époque, à l'élaboration ou à la révision de leurs propres comptabilités nationales. II conviendra à ce titre de mettre en rapport la signification et l'utilité prêtées à ces chiffres en AOF et celles qui peuvent leur être prêtées au même moment en métropole ou au sein des organisations internationales. La troisième question, liée à la précédente, porte sur le rôle politique des premiers revenus nationaux africains, derrière leur aspect technique tels qu'ils se donnent à voir (Ferguson 1990). En figurant une certaine idée du progrès, ces chiffres ont incontestablement concouru à valoriser les politiques économiques menées sous le colonialisme tardif et à orienter les politiques attendues pour atteindre le développement. Mais cela ne suppose pas pour autant un processus intentionnel et univoque de la part des multiples acteurs ayant participé à l'émergence des premiers revenus nationaux africains, comme si les uns et les autres s'étaient attachés à mettre en place une machine bien huilée : il s'agira bien au contraire de montrer les multiples décalages, désaccords et luttes de sens auxquels cette émergence a donné lieu dès les années 1950.

\section{Des richesses coloniales à la richesse nationale}

Comment mesure-t-on les richesses aux colonies avant que l'émergence du revenu national dans les années 1950 ? Même si ce n'est qu'en 1945 qu'est mis en place en AOF un service de 
la Statistique, les autorités coloniales s'attachent, dès la conquête, à "gouverner par les nombres " (Desrosières 2008b). En matière de production, chaque secteur d'activité, qu'il soit taxé de "traditionnel " ou de "moderne ", fait l'objet, depuis les années 1920, d'une comptabilisation annuelle extrêmement précise, à défaut d'être fiable, sans que nécessité soit ressentie d'une quantification globale. Là est ce que je voudrais en premier lieu montrer : l'apparition du revenu national en AOF dans les années 1950 ne résulte pas de nouvelles modalités de calcul de la production, de la mise en place de nouveaux dispositifs d'enquêtes, ni même d'une reconsidération d'un certain nombre d'activités qui auraient été auparavant ignorées; elle découle d'une nouvelle signification générale donnée à différentes statistiques productives qui jusqu'alors étaient dotées de fonctions bien différenciées et qui, après la Guerre, sont agrégées en un indice synthétique pour satisfaire une nouvelle demande politique.

\section{Mesurer les richesses avant le temps du revenu national}

Si l'on revient sur les années qui précèdent l'émergence d'un revenu national pour examiner la manière dont les services coloniaux calculent la valeur de la production en AOF, tous les secteurs ne sont pas également considérés, à l'instar de ce qui a été observé ailleurs, en particulier en Afrique britannique par Morten Jerven (2011). C'est en la matière, et dans la logique du Pacte colonial, la production destinée aux exportations qui fait office de secteur privilégié : si le Sénégal ou la Côte d'Ivoire sont plus « riches" que la Mauritanie ou que le Niger, c'est que l'une, principalement par l'arachide et l'autre, par le café et le cacao, fournissent davantage de richesses à la métropole que les deux dernières. Ces productions destinées à l'exportation font l'objet d'une comptabilisation rigoureuse, non seulement parce qu'elles figurent le rendement économique de ces différentes colonies au sein de l'Empire, mais aussi parce que c'est sur les droits d'entrée et de sortie des produits commercialisés que repose la fiscalité indirecte, laquelle fournit l'essentiel des ressources du budget fédéral. Cette mise en exergue du secteur d'exportation au sein de la production procède donc tout à la fois d'une mesure de l'apport colonial à l'économie impériale mais aussi, plus concrètement, de la gestion financière des colonies.

Outre la production destinée à l'exportation, la Direction générale des Finances dispose également de données précises sur les " exploitations à caractère industriel ", les bénéfices desquels sont également imposés. ${ }^{2}$ L'un et l'autre constituent - avec les productions assurées par les services administratifs - le secteur dit " moderne ", par opposition aux secteurs dits " traditionnels ", lesquels comportent principalement l'agriculture de subsistance, l'élevage et l'artisanat destiné aux marchés locaux. En considérant que le paradigme racial structure bien des pratiques coloniales (Reynaud-Paligot 2006), il est tentant de voir dans ce dualisme 
une représentation racialisée de la production coloniale avec, d'un côté, celle qui intéresse les métropolitains et, de l'autre, celle, dévaluée, qui ne concernerait que les indigènes. Ce serait cependant une erreur de penser que seule la première fait l'objet d'une comptabilisation précise par les services coloniaux, en tout cas pour ce qui est de l'Afrique française. La production indigène, en particulier la production vivrière, donne tout autant lieu à une élaboration régulière de chiffres depuis au moins $1926 .{ }^{3}$ Mais outre que ceux-ci entretiennent un lien plus incertain avec la réalité au vu de la faiblesse de l'appareil statistique mais aussi de bonnes raisons qu'ont les populations de se méfier des opérations comptables des administrateurs (Alpha Gado 1988, 250-255), leur usage au sein du gouvernement colonial est tout autre.

Cet usage est de deux ordres. Les statistiques vivrières ont tout d'abord une fonction idéologique : sachant que l'administration coloniale fonde une partie de son autolégitimation sur sa propension à nourrir les populations colonisées - l'idée selon laquelle les famines ont cessé avec la colonisation est un leitmotiv des discours administratifs durant les années 1930, depuis les commandants de cercle jusqu'aux ministres (Hardy et Richet 1933) - elles concourent à façonner l'image d'un État colonial grenier. II ne s'agit que d'une projection : dans les faits, ces chiffres ne parviennent absolument pas à déjouer les crises alimentaires et, lorsque celles-ci arrivent, ce n'est que rétrospectivement, à la lumière de ses manifestations concrètes, que l'administration en prend acte, réécrivant les statistiques de manière à les faire coller à la réalité empiriquement perçue (Bonnecase 2009). La seconde fonction des statistiques vivrières est fiscale, non que la production agricole soit imposée, ${ }^{4}$ mais qu'elle donne une indication sur le niveau de ressources des populations rurales. L'histoire de la colonisation est traversée par des débats relatifs au niveau de l'imposition directe - la capitation - prélevée en argent sur les collectivités. Évaluer la production indigène est un outil d'ajustement global de l'imposition, que ce soit pour la rendre moins lourde - c'est ainsi l'un des enjeux qui animent la conférence des gouverneurs généraux convoquée en 1936 sous le Front populaire, ${ }^{5}-$ ou au contraire pour l'augmenter (Ancian 1952).

Si l'administration coloniale dispose donc d'une multitude de données chiffrées pour évaluer différents pans de la production dans les années 1940, elle ne dispose pas de donnée synthétique qui relie les unes et les autres dans un même nombre. Cela ne constitue que partiellement une spécificité coloniale : il convient de relativiser cette absence d'agrégat à l'aune d'une plus large histoire des revenus nationaux. Dans les pays industrialisés, ce n'est qu'à partir des années 1930 que le revenu national commence à faire l'objet d'une comptabilisation précise par les pouvoirs publics, alors qu'émerge l'idée selon laquelle il appartient aux États de piloter l'équilibre macro-économique : selon les innovations 
théoriques formalisées par Keynes, l'économie apparaît désormais comme un grand tout articulé par quelques grands flux, lesquels demandent à être traduits en chiffres synthétiques (Desrosières 2003a). Pour chacun des pays industrialisés, on peut dater précisément cette irruption d'une statistique globale dans la gestion politique de l'économie : aux États-Unis, le Sénat préconise pour la première fois en 1934 d'établir des « estimations du revenu national » dans le contexte de la crise de 1929. En Grande-Bretagne, après des initiatives discrètes dans les années 1930, l'établissement des comptes nationaux se systématise à partir de 1941, à des fins de rationalisation de l'effort de guerre (Desrosières 2003b). En France, l'évolution est plus tardive puisque, si les initiatives planificatrices du gouvernement de Vichy vont de pair avec une tentative de rénovation de la statistique productive, ${ }^{6}$ ce $n^{\prime}$ est qu'avec la création du Commissariat du Plan en 1945, puis de l'INSEE (Institut national de la statistique et des études économiques) en 1946, qu'émerge la comptabilité nationale moderne (Fourquet 1980).

Vu sous cet angle, l'initiative de 1953 en AOF pourrait n'être lue que comme la simple déclinaison, avec sept années de décalage, de la nouvelle demande d'agrégats formulée en métropole dans le nouveau contexte keynésien d'après-guerre. Mais outre que, au regard des conditions de conception des chiffres et de leurs usages politiques, les premiers revenus nationaux de l'AOF sont assez différents de leurs homologues métropolitains (comme on le verra ultérieurement), ils apparaissent également autrement plus novateurs. Si la comptabilité nationale est nouvelle en 1945 dans la métropole, le revenu national n'en a pas moins donné lieu à de multiples évaluations depuis le dix-septième siècle ainsi que le rappelle André Vanoli (2002, 20-47). Pareilles évaluations, presque toujours d'initiative privée, étaient principalement mues par des motivations internes de réforme mais pouvaient aussi répondre à une recherche de prestige et de comparaison internationale (Studenski 1958). Cela est plus patent encore si l'on se réfère non pas au calcul des flux productifs mais à celui des patrimoines nationaux. De nombreux tableaux comparatifs en la matière sont dressés au dixneuvième siècle, alors que la statistique internationale est en plein essor (Brian 1989), pour comparer le niveau de fortune des pays touchés par l'industrialisation. Dans l'entre-deuxguerres, une comparaison est même établie dans le cadre de la Société des Nations (SDN) pour évaluer les richesses respectives des États-Unis et des principales puissances européennes (Vanoli 2002, 383). Mais les colonies ne sont pas conviées dans cette comparabilité internationale, laquelle reste "géographiquement limitée aux seuls pays civilisés » (Horvath 1972, 289).

L'émergence d'un revenu national en AOF, tout en s'inscrivant dans un mouvement plus général de valorisation des agrégats économiques dans le monde au sortir de la Deuxième Guerre mondiale, traduit donc quelque chose de plus spécifiquement nouveau dans un 
contexte colonial : la possibilité de placer les colonies, par le chiffre, dans un même continuum que les pays non colonisés et de les comparer à ces derniers.

\section{Une nouvelle demande d'indicateur global}

II s'agit dès lors de s'interroger sur les raisons de cette innovation statistique ou, plus précisément, sur les nouvelles demandes politiques à laquelle elle répond. Deux facteurs principaux, liés l'un à l'autre parce qu'ils s'inscrivent l'un et l'autre dans le nouveau contexte développementaliste de l'après-guerre, peuvent être invoqués. Le premier, déjà évoqué, est endogène et relève de la mise en place de la nouvelle politique volontariste d'investissements coloniale. Le second, exogène, réside dans la nouvelle demande internationale d'indicateurs à des fins de comparaison internationale et de mesure du développement.

En premier lieu, ce n'est pas un hasard si le premier service de statistique créé pour 1'AOF en 1945 se prénomme service de la Statistique et du Plan : c'est le gonflement des investissements publics et la nouvelle politique de planification mise en place dans le cadre du nouveau Fonds d'investissement pour le développement économique et social (FIDES) qui suscitent une nouvelle demande statistique, notamment sur la production. On retrouve le même phénomène en Afrique britannique où les premières études du revenu national, à commencer par celle menée en 1948 par Phyllis Deane (1948) pour la Rhodésie du Nord, sont sous-tendues par la nouvelle politique d'investissements mise en place au début de la décennie à la suite du Welfare and Development Act de 1940 (Cooper 2004, 40-41). Idéalement - c'est-à-dire, selon la logique qui prédomine au même moment dans les comptabilités nationales métropolitaines - il appartiendrait désormais aux statisticiens de faire le bilan des ressources disponibles dans les colonies, mais aussi d'en prévoir les différentes attributions. En pratique, la chose est plus malaisée dans le cadre de l'AOF ainsi que l'exprime la Direction générale des Finances dans son rapport de 1950 :

Si la perfection sollicite qu'il soit établi un bilan des ressources du pays et qu'ensuite, en fonction d'une politique déterminée, l'autorité fixe la part de ces ressources à attribuer à la consommation privée, à la consommation publique et au développement du capital de l'AOF, il est bien difficile, en l'état actuel des renseignements statistiques et des moyens d'investigations du pays, de faire autre chose que de l'empirisme légèrement corrigé par quelques observations de base.7

Sont ici énumérés les trois éléments qui fondent, en métropole, le calcul du revenu national en fonction de la demande finale (la consommation, la dépense publique et les investissements), laquelle est précisément privilégiée pour calculer le revenu national dans le cadre des nouvelles politiques keynésiennes (Desrosières 1994, 384). Or, s'il apparaît difficile, 
au tout début des années 1950, de transposer une telle démarche en AOF, c'est pour des raisons de faisabilité pratique, mais aussi parce que son utilité n'apparaît pas aussi évidente.

Plutôt qu'à planifier l'attribution à venir des ressources disponibles, le calcul d'un revenu national répond, dans l'optique de la Direction générale des Finances, à deux préoccupations plus immédiates. Le premier, classique comme on l'a vu, réside dans l'évaluation de la ponction fiscale. Pareille préoccupation est ancienne : dans les rapports financiers des années 1940, il est régulièrement question de mesurer « l'effort fiscal » des différentes colonies; mais à l'époque, le niveau d'imposition est rapporté, faute d'autre référent, à la population dans son ensemble. ${ }^{8}$ Avec l'évaluation du revenu national, il devient possible à «la puissance publique d'avoir au moins une idée de la ponction qu'elle prélève par l'impôt sur [le] produit » et de s'assurer que " celle-ci a atteint sa limite $" .^{9}$ La deuxième préoccupation consiste, plus qu'à planifier par avance l'attribution des ressources disponibles, à mesurer après coup la croissance économique induite par les investissements, partant du principe, selon les termes de la Direction générale des Finances de l'AOF en 1953, que « l'on ne peut valablement parler d'accroître un revenu dont on ne connaît pas au moins l'ordre de grandeur $"{ }^{10}$ Cette attente est clairement exprimée deux ans plus tard par la Direction des Affaires économiques et du Plan du ministère de la France d'outre-mer:

Les données fragmentaire dont nous disposons laissent deviner une très large expansion de la richesse publique et une élévation rapide du niveau de vie dans les territoires d'outre-mer depuis la mise en application du premier programme du plan de l'après-guerre, [...] mais il serait plus démonstratif encore d'accuser la signification de ces indices de l'activité économique en dégageant de leur ensemble et de la masse des autres données numériques existantes un chiffre unique pour chaque territoire et pour chaque année, celui qui traduirait ce qu'on appelle ordinairement, dans les pays d'Europe, le revenu national. ${ }^{11}$

Ainsi, plus qu'un outil de planification et de prévision des politiques publiques, selon la logique qui prévaut au même moment en métropole, le calcul du revenu national dans les colonies traduit la volonté gouvernementale d'évaluer les effets induits par la nouvelle politique d'investissement coloniale sur la production locale et les conditions de vie des populations colonisées. Pareille optique demande à être replacée dans la profonde crise de légitimité qui affecte l'Empire colonial français dans l'après-guerre : sans qu'il soit nécessairement dans l'intention des premiers concepteurs des revenus nationaux de répondre directement à cette crise, leurs travaux n'en ouvrent pas moins la possibilité de prouver par le chiffre, pour la première fois dans l'histoire de la colonisation, l'impact positif de la politique coloniale sur la richesse globale des colonies et de ses habitants. C'est d'ailleurs ce qu'en retiendront, après les Indépendances, les statisticiens français chargés de mettre en place les comptabilités 
nationales des nouveaux États africains, cette fois à des fins affichées de planifications des politiques de développement :

Les [premiers] travaux relatifs au produit intérieur brut [...], quand ils furent entrepris, avaient pour objet essentiel d'apprécier le niveau de vie en Afrique de l'Ouest. [...] Par le détour du produit intérieur, on cherchait en fait à appréhender les utilisations finales intérieures - ou plus exactement leurs contreparties en termes de revenus. (Haubert 1969, 43-44)

En second lieu, cette quête de légitimation par les chiffres est également sous-tendue, de manière exogène, par une demande internationale. L'une des principales activités des nouvelles organisations onusiennes mises en place à partir de 1945 consiste à produire une standardisation des savoirs à des fins de comparabilité internationale d'une part et de mesurabilité du développement d'autre part. Chaque organisme spécialisé agit en ce sens dans son propre domaine : la FAO (Food and Agriculture Organization) par exemple, s'attache tout à la fois à standardiser la collecte de données sur la production agricole dans le cadre de grands recensements décennaux mondiaux, et à produire de nouvelles normes de besoins alimentaires minima à l'aune desquelles mesurer et comparer les niveaux d'alimentation dans le monde. Cette démarche s'inscrit dans la lignée d'une dynamique déjà à l'œuvre dans l'entre-deux-guerres sous l'égide de la SDN mais qui, pour l'essentiel, ne concernait que les pays européens et nord-américains. L'une des grandes préoccupations onusiennes de l'immédiat après-guerre consiste à inclure les territoires désormais « sous-développés » dans l'espace de comparabilité induit par la statistique internationale, rompant ainsi la barrière d'incommensurabilité qui séparait jusqu'alors les colonies et les métropoles (Bonnecase 2011) : sous le prisme du développement, les conditions de vie dans le monde sont désormais posées comme étant tout à la fois mesurables, comparables et rattrapables.

L'une des questions qui agitent les instances internationales résident alors dans la question des critères adéquats : sachant que la Charte des Nations unies proclame que l'activité économique et sociale internationale doit tendre à instaurer « de meilleures conditions de vie $"{ }^{12}$ il s'agit tout à la fois de préciser ce que recouvrent cette notion et les méthodes qui doivent permettre de mesurer son élévation. En mai 1949, l'Assemblée générale adopte ainsi une résolution demandant au Conseil économique et social d'examiner « la situation sociale dans le monde » à partir " d'indices quantitatifs de satisfaction de besoins dont l'existence est universellement reconnue $» .^{13}$

Trois ans plus tard, le Conseil économique et social établit un premier « rapport sur la situation sociale dans le monde et les niveaux de vie en particulier » dans lequel "le revenu national par habitant " est " considéré comme un indice des niveaux de vie ", même si " on ne s'est pas encore nettement mis d'accord sur ce qu'il fallait entendre au point de vue international 
par "niveau de vie" ". ${ }^{14}$ Parallèlement une nouvelle résolution de l'Assemblée générale des Nations unies prie le Conseil économique et social de " faire élaborer des méthodes et des techniques statistiques appropriées de manière à faciliter au maximum le rassemblement et l'emploi de données pertinentes afin que le Secrétariat général puisse publier régulièrement des rapports annuels indiquant, en chiffres absolus, les changements intervenus dans tous les pays en ce qui concerne les conditions de vie $"{ }^{15}$

Suite à cette résolution, un comité de six experts est réuni en juin 1953 par le Conseil économique et social afin de travailler à « la définition et l'évaluation des niveaux de vie du point de vue international $»{ }^{16}$ Des membres de l'OIT (Organisation international du travail), de I'UNESCO (Organisation des Nations unies pour l'éducation, la science et la culture), de la FAO et de l'OMS (Organisation mondiale de la santé) participent également aux réunions de travail. Le comité s'appuie en outre sur les avis formulés par une trentaine d'universitaires, de ministres ou d'anciens ministres, majoritairement d'Europe et des États-Unis, plus marginalement d'Asie du Sud ou d'Amérique latine. ${ }^{17}$ Outre le bienfondé de la démarche dans son ensemble et le "risque de préjugé ethnocentrique " qu'elle comporte - parce que « l'emploi d'un système de valeurs uniforme et universel pourrait conduire à « sanctionner sur le plan international les progrès techniques réalisés dans l'Occident » -, l'une des principales controverses touche à la pertinence d'un « indice unique du niveau de vie global ». Le revenu national apparaît à cet égard comme " un indicateur relativement complet » tout en pouvant susciter des « conclusions assez fausses sur les différences qui existent entre les niveaux de vie des diverses régions du monde ", d'une part parce qu'il est difficile de « mesurer la parité de pouvoir d'achat et [de] convertir les monnaies nationales ", d'autre part parce qu'une " partie des biens et services ne relèvent pas de la consommation marchande ». En revanche, le comité cible la " variation du revenu national par habitant " comme étant l'un des principaux indicateurs permettant de mesurer "l'évolution du niveau de vie général » d'une population dans un même pays, à défaut de permettre des comparaisons internationales pertinentes. ${ }^{18}$ On retrouve là des débats par la suite maintes fois repris, parfois dans une prétention novatrice, au sein des organisations onusiennes, des instances académiques ou des médias internationaux et qui, finalement, ont d'emblée fait partie de I'histoire du revenu national comme indicateur de niveau de vie. ${ }^{19}$

Deux choses importantes ressortent de ce retour sur les conditions d'émergence du revenu national en AOF. D'une part, cette émergence constitue une rupture dans l'appréciation des richesses coloniales en ce qu'elle ne connaît pas de précédents comparables aux prémices de la comptabilité nationale en métropole. D'autre part, elle répond à des demandes hétérogènes où se mêlent des considérations internes anciennes - en particulier ce qui touche 
à la fiscalité - et un nouveau souci de mesurabilité des niveaux de vie dans le monde. II paraît important d'insister sur le fait que le revenu national n'a pas été doté après coup de cette dernière signification, ainsi que le suggère Alain Desrosières (2012) à propos des seuls pays industrialisés : en Afrique tout au moins, cette valeur d'indicateur de niveau de vie, aussi controversée soit-elle, a été présente au moment même de l'élaboration des premiers revenus nationaux.

\section{Quels chiffres et pour quoi faire?}

Je voudrais maintenant interroger les conditions concrètes d'élaboration des premiers revenus nationaux en AOF et les usages qui ont pu être les leurs à la toute fin de la période coloniale. Si un terme permet de relier l'un et l'autre des deux questionnements, c'est celui de décalage : d'un côté, le calcul des premiers revenus nationaux donne lieu à de nombreuses controverses, tant relatives aux méthodes employées qu'à la fiabilité des résultats obtenus ; de l'autre, cela ne les empêche nullement de valoir comme preuves et d'être politiquement investis, parfois de manière antagonique, notamment à des fins de valorisation ou de contestation de la politique coloniale.

\section{L'incertitude des chiffres, des méthodes et de leurs concepteurs}

Les deux premières études du revenu national en Afrique française, hormis deux précédents en 1952 pour les seuls territoires du Cameroun et de Mauritanie, ${ }^{20}$ sont menées en 1953 et en 1955. La première est un « Essai de récapitulation des éléments connus à Dakar pour servir à un calcul du revenu national ", établi par un administrateur colonial, André Auclert, pour le compte de la Direction générale des Finances de l'AOF. ${ }^{21} \|$ s'agit d'une compilation des données productives disponibles, lesquelles sont converties en valeur monétaire et synthétisées en un « revenu par tête " pour l'AOF et chacune des colonies qui composent la fédération. Celle-ci se retrouve ainsi, pour la première fois de son histoire, inscrite dans une hiérarchisation internationale des niveaux de vie en fonction du revenu national (Tableau 1). La deuxième s'inscrit dans un plus large «Essai de détermination du revenu national des principaux territoires d'outre-mer ", mené sous la direction d'un administrateur de l'INSEE, Boris Maldant. Outre l'AOF, sont évaluées les productions respectives de l'Afrique équatoriale française (AEF), du Cameroun et de Madagascar pour les années 1947 et $1953 .{ }^{22}$ Ce dernier choix n'est pas fortuit puisqu'il s'agit des limites du premier plan de développement mis en œuvre dans le cadre du FIDES, dont il s'agit ainsi d'évaluer les effets sur la richesse globale de ces territoires. 


\begin{tabular}{|c|c|}
\hline Territoire & Revenu par tête en francs CFA \\
\hline Arabie Saoudite (1949) & 5250 \\
\hline Yémen (1949) & 5250 \\
\hline Éthiopie (1949) & 6650 \\
\hline Afghanistan (1949) & 8750 \\
\hline Pakistan (1949) & 8925 \\
\hline AOF (1951) & 9400 \\
\hline Inde (1949) & 9450 \\
\hline Bolivie (1953) & 10000 \\
\hline Pérou (1949) & 11130 \\
\hline Argentine (1948) & 15240 \\
\hline Brésil (1950) & 22810 \\
\hline Cuba (1950) & 55125 \\
\hline
\end{tabular}

Tableau 1 - Quelques revenus nationaux dans le monde (1948-1951)

(Source : BNF, Gouvernement général de l'AOF, Direction générale des Finances, « Essai de récapitulation des éléments connus à Dakar pour servir à un calcul du revenu national de l'AOF en 1951 », Dakar, 1953)

L'une et l'autre de ces deux études sont significatives des controverses méthodologiques et, finalement, des incertitudes qui marquent l'établissement des premiers revenus nationaux dans les colonies africaines. L'une des principales interrogations porte sur les «termes récalcitrants ", autrement dit les secteurs productifs dont la mesure pose le plus problème, soit qu'ils soient non marchands, soit que leur qualité même de "production " prête à contestation. Apparaissent ainsi principalement comme "récalcitrants " dans les colonies africaines l'autoconsommation paysanne, les services rendus par les ménages à eux-mêmes et les logements occupés par leurs propres propriétaires. La prise en compte de chacun de ces termes dans le calcul du revenu national donne lieu à des débats qui dépassent les limites de l'Empire français, s'inscrivant ainsi dans les nouvelles formes de connexions inter-impériales qui se dessinent après la Guerre (Kent 1992). Concernant l'autoconsommation paysanne, se posent à la fois le problème de son évaluation quantitative et celui de sa transcription en valeur marchande.

Si ces questions, derrière leurs aspects techniques, renvoient à des enjeux éminemment politiques, il ne semble pas que cette économie domestique ait été sciemment dépréciée par les premiers concepteurs des revenus nationaux en Afrique française pour des raisons idéologiques, à l'instar de ce qu'observe Morten Jerven (2011) à propos de l'Afrique britannique : on verra à ce titre que les services coloniaux avait plutôt intérêt à présenter les 
chiffres de PIB les plus hauts possibles - économie domestique inclue - ne serait-ce que pour valoriser leur propre politique de développement. La valeur à donner aux services rendus par les ménages à eux-mêmes fait également débat : alors qu'une partie des premiers investigateurs supposent que ces services sont particulièrement importants en Afrique - dans la mesure où de nombreuses activités telles que le puisage de l'eau, la coupe du bois ou la transformation des céréales, fournies par des professionnels en Europe, sont assurées par les ménages dans les territoires africains ${ }^{23}$-, telle $n^{\prime}$ est pas la position retenue par André Auclerc et Boris Maldant, d'une part parce que « la quasi-totalité des femmes [africaines] concourent à la production agricole mesurable " et d'autre part "parce que les besognes ménagères constituent des services relativement sommaires ${ }^{24}$. Concernant enfin l'évaluation du revenu que rapporte à son propriétaire la possession d'un logement qu'il habite et pour lequel il ne paye pas de loyer (ce qui est le cas de la totalité des populations rurales), André Auclerc s'inspire des études faites par Phyllis Deane (1948) sur « le revenu des cases » en Rhodésie du Nord, pour en extrapoler les conclusions à l'AOF, en attendant « une enquête aussi localisée que possible afin de connaître le prix de revient de ce type de logement » dans la fédération. ${ }^{25}$

Une seconde interrogation touche, non pas aux méthodes de calcul, mais aux sources empiriques sur lesquelles reposent un certain nombre de données collectées, en particulier celles qui ont trait aux cultures vivrières, à la production pastorale et à la population. Pour ce qui est d'abord des cultures vivrières, les chiffres reposent ainsi, faute d'enquête par sondage, ${ }^{26}$ sur l'estimation annuelle des rendements et des surfaces ensemencées par les agents des services agricoles. Sachant que ces derniers sont presque toujours amenés à extrapoler à l'ensemble des circonscriptions qu'ils ont à charge leurs observations effectuées dans quelques villages, les résultats restent de l'aveu du service de la Statistique de l'AOF assez aléatoires. L'évaluation des produits de l'élevage, ensuite, repose sur les recensements pastoraux à finalité fiscale, lesquels sous-estiment invariablement la réalité du fait des stratégies d'évitement développées par les éleveurs à l'égard des agents recenseurs. Pour pallier cette imperfection, les statisticiens établissent un « coefficient de sous-déclaration fiscale ${ }^{27}$ destiné à redresser la valeur de la production pastorale. La comptabilisation des populations, enfin, repose sur les recensements administratifs, lesquels visent d'abord à calculer le montant de l'impôt de capitation. Outre que les agents recenseurs ont tendance, dans ces conditions, à comptabiliser avec moins de zèle les classes d'âge non imposables, ils font eux-aussi l'objet de stratégies d'évitement qui visent à faire baisser les charges fiscales pesant sur les collectivités. Lors des premières enquêtes démographiques par sondage réalisées en Afrique francophone à partir de 1955, il sera ainsi fréquent de voir la population réévaluée de plus d'un dixième par rapport aux estimations administratives. ${ }^{28}$ 
Ces questionnements amènent finalement les statisticiens de la France d'Outre-mer à considérer avec prudence leurs premiers résultats et les conclusions que l'on pourrait en tirer, en matière d'accroissement de la richesse et de comparaison internationale. D'après l'étude publiée par le ministère en 1955 - la première à faire l'objet d'une diffusion par les services de la France d'outre-mer29 -, le revenu national s'élève en 1953 à 13600 francs CFA par habitant en AEF, à 13800 francs en AOF, à 18100 francs au Cameroun et à 23400 francs à Madagascar et présente des taux de croissance globale compris entre 50 pour cent et 80 pour cent par rapport à 1947, année de lancement du premier plan du FIDES (Tableau 2). Les auteurs de l'étude prennent toutefois soin de conclure dans ces termes :

Ces récapitulations ne doivent [...] pas être considérées comme une évaluation définitive du revenu national des territoires, et méritent de sérieuses réserves dans leur état actuel, car elles sont entachées de sous-estimations de toute sorte. [...] Une particularité remarquable [...] est l'alignement de la production par habitant de l'AEF sur le taux de l'AOF. Une telle constatation va à l'encontre des données classiques. [...] II est probable que sur le plan des niveaux de vie véritables, l'AOF devrait être créditée de 1000 à 1500 francs de plus [...] relativement à l'AEF. [...] On peut toutefois se demander si, réellement, les deux territoires n'ont pas des standings plus voisins qu'on le croit. $^{30}$

\begin{tabular}{|c|c|c|c|c|}
\cline { 2 - 5 } \multicolumn{1}{c|}{} & AEF & AOF & Cameroun & Madagascar \\
\hline PNB 1947 (FCFA*) & 50500000 & 160300000 & 37300000 & 72400000 \\
\hline PNB 1953 (FCFA) & 71300000 & 271800000 & 67000000 & 110800000 \\
\hline Croissance 1947-1953 & $+41 \%$ & $+69 \%$ & $+80 \%$ & $+53 \%$ \\
\hline PNB par habitant 1953 (FCFA) & 13600 & 13800 & 18100 & 23400 \\
\hline
\end{tabular}

* FCFA de 1953

Tableau 2 - Estimations du PNB en Afrique française (1947-1953)

(Source : CAOM, 1fides/69, ministère de la France d'outre-mer, « Essai de détermination du revenu national des principaux territoires d'outre-mer en 1947 et en $1953 », 1955)$

Deux éléments ressortent de cet examen des conditions d'élaboration des premiers revenus nationaux en AOF. D'une part, les statisticiens chargés de leur élaboration, tout en répondant à des préoccupations proprement productives dans le cadre de la planification coloniale, attribuent également à leurs chiffres une valeur de mesure des "niveaux de vie", conformément à ce qui a été observé à l'échelle internationale. D'autre part, ces chiffres peinent à faire autorité face à la réalité telle qu'elle est empiriquement perçue : I'alignement 
statistique de l'AOF sur le niveau de l'AEF apparaît ainsi éminemment suspect au regard des perceptions habituelles de la chose, à tel point que les statisticiens puissent inviter à se demander, au terme de leur étude, ce qu'il en est réellement.

\section{Dire des vérités par les chiffres}

Or, ces réserves n'empêchent nullement ces chiffres, une fois établis, d'échapper à leurs propres concepteurs, de valoir comme preuves et de signifier des vérités. Là est sur ce quoi je voudrais finir : les premiers revenus nationaux établis en AOF ont beau être quelque peu incertains, ils furent rapidement investis politiquement et cela, à des fins assez contrastées, voire parfois antagoniques. S'il n'est pas question de saisir ici la totalité des débats au sein desquels ont figuré ces indicateurs dans les années 1950, je voudrais juste cibler quatre usages qui en ont été faits.

Le premier usage, le plus prévisible puisque là était l'attendu de départ, réside dans la valorisation de la nouvelle politique d'investissements coloniale par le ministère de la France d'outremer. Dans ce cas, plus que le revenu national lui-même, c'est son augmentation qui fait office d'indicateur privilégié. De 1946 à 1953, durant la mise en œuvre du premier plan du FIDES, cette augmentation atteindrait près de 9 pour cent par an en AOF. Cela permet aux statisticiens du ministère de conclure prudemment à un rôle positif du plan, lequel est « le seul élément nouveau d'après-guerre qui ait exercé une action assez marquée et assez continue pour rendre compte de la rapide expansion des revenus territoriaux en l'espace de ces six années $» .31$ Pareil bilan comptable est en revanche relayé sans distance par une presse coloniale qui se félicite, revenus nationaux à l'appui, que « grâce à l'effort français, les niveaux de vie se [soient] élevés dans les colonies ".32 Ces chiffres, que l'on peut également retrouver dans des livres d'histoire sur la colonisation (Ageron 1990, t.2, 453), concourent finalement, aux côtés d'autres statistiques, notamment médicales, à présenter le colonialisme tardif sous un jour favorable, celui du " renouveau » et des " réalisations tardives » (Bado 1996, 349). II ne s'agit pas ici de nier le fait que les investissements français de l'après-guerre aient été synonymes d'accroissement de la production - et il n'est pas entré dans ma démarche de vouloir substituer aux statistiques officielles d'autres chiffres qui seraient les vrais chiffres de la croissance. Il s'agit juste de bien voir que les premiers revenus nationaux de l'AOF ont permis de figurer un succès malgré les incertitudes qui ont marqué leurs modalités d'élaboration.

Un deuxième usage, plus synchronique et davantage tourné vers les attentes internationales, consiste à inscrire les chiffres du revenu national sur une échelle de développement afin de mesurer la position relative de l'AOF par rapport à d'autres territoires. Un tel usage va là 
encore à l'encontre des réserves affichées par les statisticiens pour lesquels, si « la notion de niveau de vie moyen peut être dégagé en divisant le revenu national par le nombre d'habitants ", le procédé reste " très critiquable en matière de comparaison internationale " (Fabre, Dubois, et Courcier 1958, 7). Pareil usage est en outre ambivalent puisque, selon le point de vue où l'on se place et les comparaisons que l'on mobilise, il est possible d'en tirer des conclusions plus ou moins favorables à la politique coloniale de la France. Cette ambivalence est perceptible dès 1953, alors que la Direction générale des Finances de l'AOF, munie de son premier revenu national, entreprend de dresser un tableau comparatif des niveaux de développement dans le monde (Tableau 1). D'un côté, elle admet que « le revenu par tête de l'AOF » est " caractéristique d'un pays sous-développé ». Mais de l'autre, elle met en exergue le fait que " des nations dites libres, devenues des accusateurs internationaux, sont également sous-développées, et souvent d'une manière plus grave ". II apparaît ainsi utile à la Direction générale " de rappeler quelles sont les estimations connues du revenu national dans quelques pays sous-développés du monde parmi lesquels on trouve les plus farouches de nos détracteurs dans les assemblées internationales $॥ .33$ Or, il est possible, à partir du même indicateur, d'aller chercher des conclusions opposées.

Un troisième usage, fait des revenus nationaux dans les années 1950, consiste ainsi à mettre en cause l'efficacité de la politique coloniale française en termes d'élévation des niveaux de vie. L'un des principaux courants critiques des investissements métropolitains dans les colonies est connu : il s'agit du cartiérisme, du nom du journaliste Raymond Cartier qui, dans trois articles publiés par Paris Match en août et septembre 1956, met en cause la nouvelle politique coloniale d'après-guerre. Ce qui est moins connu, c'est la manière dont ce discours mobilise les propres chiffres de l'administration coloniale pour les retourner contre elle. Ainsi, selon Cartier :

En Haute-Volta, on touche aux niveaux de vie les plus bas de l'Afrique française. Le revenu moyen du paysan mossi est évalué à 5000 francs CFA, soit 10000 francs métropolitains par an. Sur les marchés, les femmes vendent des œufs microscopiques et des poignées d'arachides que l'on paye avec des vieilles pièces en bronze d'aluminium d'un ou deux francs. C'est pour le commun de ces pauvres gens qu'on croit nécessaire d'édifier des halles en béton, hautes comme une cathédrale ! [...] Malgré la Côte d'Ivoire, qui relève la moyenne en se rapprochant du bien-être de l'Espagne, le niveau de vie de l'AOF ne dépasse pas celui de l'Inde, et il tombe, en Haute-Volta, à l'étage du Yémen. (Cartier 1956)

Ces assertions - jusqu'aux exemples invoqués - ne sont rien d'autre que la mise en mots du tableau statistique établi par la Direction générale des Finances de l'AOF trois ans plus tôt. Cela illustre finalement la manière dont le débat public métropolitain s'empara assez rapidement du revenu national et de la quantification des niveaux de vie pour en faire un 
argument global, à charge ou à décharge, de la politique coloniale française en Afrique. Or, il convient en dernier lieu d'interroger l'usage de ces chiffres par les premiers destinataires de cette politique, en l'occurrence les populations colonisées. Là encore plusieurs directions différentes ont pu être prises dans les discours des syndicats et des partis africains durant les dernières années de la colonisation. Si l'on se focalise d'abord sur l'écart entre les colonies et la métropole, le revenu national par habitant peut d'abord être invoqué pour signifier une inégalité foncière. Mais il ne me semble pas que cet usage des chiffres soit répandu entre 1953 et l'indépendance. Dans les plateformes des principales formations politiques interterritoriales constituées en AOF, ce sont d'autres indicateurs - en particulier le taux de mortalité infantile ou celui de la ration calorique - qui font office d'arme statistique en matière de comparaison avec la métropole.34 Si l'on se focalise ensuite sur la structure interne des revenus nationaux, les nouveaux savoirs permettent de quantifier des écarts entre différentes populations qui, jusqu'alors, n'avaient jamais été mesurés : il peut être ainsi affirmé, à la fin de la décennie, que « le niveau de vie » dans la fédération est en moyenne près de 40 fois plus élevé chez "les blancs " que chez " les noirs", en admettant que "les revenus des secteurs modernes échoient principalement aux premiers " tandis que ceux des "secteurs mixte et traditionnel » reviennent aux seconds (Capet et Fabre 1957, 13). Si l'on se focalise enfin sur l'augmentation de la production dans les années 1950, le revenu national peut être invoqué pour négocier de meilleurs traitements, notamment en terme salarial, avec l'administration. Là est davantage la position des syndicats de la fédération qui, tout en indexant une augmentation du coût de la vie, arguent de la croissance économique - et des bénéfices des entreprises - pour exiger l'augmentation des salaires. 35 En tout état de cause, cela invite aussi à voir dans l'émergence des revenus nationaux à la fin de la période coloniale une nouvelle opportunité discursive pour les acteurs politiques africains.

\section{Conclusion}

Trois éléments, qui se dégagent de cette histoire des premiers revenus nationaux en AOF, peuvent être mis en perspective à la lumière de travaux récents. Premier élément, ces chiffres sont incertains au regard de leurs conditions de production. Cela ne constitue que moyennement une surprise : plusieurs auteurs, et essentiellement Morten Jerven pour I'Afrique anglophone, ont déjà montré que les chiffres de la croissance pouvaient parfois se réduire à de simples jeux d'écriture, soit qu'il y ait faiblesse de l'appareil statistique, soit qu'il y ait changement dans les modalités de calcul ou même, parfois, volonté délibérée de travestissement (Jerven 2009, 2010). Ces jeux sur les chiffres constituent en outre nullement une réalité spécifiquement africaine ni même spécifiquement coloniale, pas plus qu'ils ne se cantonnent à un passé révolu (Data 2009). L'histoire que j'ai ici racontée est finalement 
relativement banale : elle traduit la propension de chiffres, une fois établis, à poursuivre leur propre histoire, indépendamment de leurs propres concepteurs et à revêtir des significations que ces derniers n'y avaient pas nécessairement mis. Second élément, ces chiffres ont eu une place importante dans la justification tardive de la colonisation, à un moment où celle-ci souffrait d'un déficit marqué de légitimité. Cela incite à lire les revenus nationaux, non pas seulement comme des outils descriptifs, même imparfaits, mais aussi comme des biais de gouvernement et de légitimation. C'est ce que fait Béatrice Hibou (2011) à propos du cas tunisien, lorsqu'elle lit les indicateurs macro-économiques comme les composants d'un récit statistique - celui du " miracle » - lequel était devenu central dans l'appareil idéologique de l'État benaliste et la fabrique du consentement. Sans vouloir transférer au colonialisme tardif les caractéristiques de ce régime autoritaire, tout au moins peut-on affirmer que les premiers chiffres du revenu national s'inscrivent également dans un récit statistique, celui du succès tardif de la politique économique de la colonisation, dont on peut se demander s'il n'a pas, plus tard et par contraste, contribué à écrire un autre récit, celui de la faible croissance des pays africains après les indépendances. Troisième élément, le revenu national a fait office, dès les années 1950, d'indicateur des niveaux de vie en Afrique sans que ce soit son seul usage, ni que cet usage-là soit autant publicisé qu'il ne le sera par la suite. II ne me semble pas, à ce titre, que l'on puisse clairement distinguer, ainsi que le fait Alain Desrosières (2008b, 18-19) sur la base d'observations européennes et américaines, un premier usage expert du revenu national focalisé sur sa signification macroéconomique et un usage grand public plus récent qui ferait du revenu national un indicateur de richesse. Dès les années 1950 - et pour ce qui est de l'Afrique -, l'un et l'autre de ces deux usages se sont d'emblée mélangés et la propension du revenu national à figurer le niveau de vie a fait l'objet de controverses, dont on peut observer les prolongements jusqu'à aujourd'hui.

\section{Notice biographique}

Vincent Bonnecase est docteur en histoire et chercheur en science politique au Centre national de la recherche scientifique à Bordeaux (France). Il a travaillé sur I'histoire de la mesure de la pauvreté en Afrique coloniale et postcoloniale. Il a notamment écrit La Pauvreté au Sahel. Du savoir colonial à la mesure internationale (Paris, Karthala, 2011). Il travaille désormais sur les mobilisations contre la vie chère en Afrique contemporaine.

\section{Notes}

1. L'AOF est l'une des deux grandes fédérations coloniales détenues par la France en Afrique. Établie en 1895, elle regroupe le Sénégal, la Mauritanie, le Soudan français (Mali), le Niger, la Guinée, la HauteVolta (Burkina Faso), la Côte d'Ivoire et le Dahomey (Bénin). 
2. Archives nationales du Sénégal (ANS), 2G50.40, Contrôle financier de l'AOF, « Rapport sur les budgets de l'AOF », Dakar, 1950.

3. C'est à partir de 1926 que les services de l'Agriculture de l'AOF doivent, par arrêt du gouvernement général, comptabiliser le volume de la production agricole chaque année dans chaque colonie.

4. Il existe toutefois une taxe sur le bétail.

5. ANS, 17G255, ministère des Colonies, "Rapport sur la Conférence des gouverneurs généraux », Paris, 1936.

6. La loi du 6 avril 1941 portant sur le plan d'équipement national prévoyait un plan d'investissement de 10 ans dans les colonies.

7. ANS, 2G50.40, Direction du Contrôle financier de l'AOF, « Rapports sur les budgets de l'AOF », Dakar, 1950.

8. ANS, 2G48.105, Direction du Contrôle financier de l'AOF, « Rapport sur la situation budgétaire de I'AOF au 30 juin 1948 », Dakar, 1949.

9. Centre des Archives d'outre-mer (CAOM), BIB-SOM, Direction générale des Finances de l'AOF, "Essai de récapitulation des éléments connus à Dakar pour servir à un calcul du revenu national de I'AOF », Dakar, 1953.

10. Centre des Archives d'outre-mer (CAOM), BIB-SOM, Direction générale des Finances de l'AOF, « Essai de récapitulation des éléments connus à Dakar pour servir à un calcul du revenu national de I'AOF », Dakar, 1953.

11. CAOM, 1fides/69, Direction des Affaires économiques et du Plan du ministère de la France d'outremer, "Essai de détermination du revenu national des principaux territoires d'outre-mer (AOF, Cameroun, AEF, Madagascar) en 1947 et en 1953 », Paris, 1955.

12. ONU (Organisation des Nations unies), Chartre des Nations unies, San Francisco, 1945, préambule.

13. ONU, Assemblée générale, résolution n²80-III du 13 mai 1949.

14. ONU, Conseil économique et social, « Rapport préliminaire sur la situation sociale dans le monde et les niveaux de vie en particulier », New York, 1952.

15. ONU, Assemblée générale, résolution n 527-VI du 26 janvier 1952.

16. Il comprend Raymond Firth et Philip Hauser, respectivement professeurs d'anthropologie en Angleterre et de sociologie aux États-Unis, Erland Hofsten et Alexander Moraes, chefs de service statistique en Suède et aux États-Unis, M. V. Rao, directeur d'un Institut d'Économie politique en Inde, et Louis- Joseph Lebret, directeur de la revue Économie et humanisme en France. 
17. Parmi les experts sollicités, on peut trouver, outre plusieurs universitaires aux États-Unis, des personnalités aussi diverses que Sumitro Djojohadikusomo, ministre des Finances de l'Indonésie, Henri Labouret, ancien administrateur colonial français, et Josué de Castro, auteur brésilien du premier ouvrage nommément consacré à la faim dans le monde (Castro 1951).

18. ONU, Conseil économique et social, « Rapport sur la définition et l'évaluation des niveaux de vie du point de vue international », New York, 1954.

19. Pour une résurgence récente et fortement médiatisée de ces débats, voir Fitoussi, Sen, et Stiglitz (2009).

20. Ces deux études menées sous la direction de deux administrateurs de l'INSEE - Jules Leveugle pour le Cameroun et Louis Marciniak pour la Mauritanie - se focalisent davantage, voire exclusivement dans le premier cas, sur la production marchande (Fabre, Dubois, et Courcier 1958).

21. CAOM, BIB-SOM, Direction générale des Finances de l'AOF, «Essai de récapitulation des éléments connus à Dakar pour servir à un calcul du revenu national de l'AOF », Dakar, 1953.

22. CAOM, 1fides/69, Direction des Affaires économiques et du Plan du ministère de la France d'outremer, «Essai de détermination du revenu national des principaux territoires d'outre-mer (AOF, Cameroun, AEF, Madagascar) en 1947 et en 1953 », Paris, 1955.

23. C'est notamment le point de vue défendu par Jules Leveugle (1953).

24. CAOM, BIB-SOM, Direction générale des Finances de l'AOF, «Essai de récapitulation des éléments connus à Dakar pour servir à un calcul du revenu national de l'AOF », Dakar, 1953.

25. Ibid.

26. Hormis quelques précédents au début de la décennie, les premières grandes enquêtes par sondage sur la production agricole et, plus largement, sur les niveaux de vie des populations sont menées en 1954-1955 en Côte d'Ivoire ("Enquête sur la subdivision de Bongouanou ») et en 1956-1958 au Sénégal ("Mission socio-économique du Sénégal») et au Soudan français ("Mission socioéconomique du Soudan ») (Bonnecase 2011).

27. Ce coefficient de sous-déclaration est lui-même calculé en recoupant les données des recensements et celles des campagnes anti-épizootiques, supposées plus conformes à la réalité, dans les zones où ces deux opérations sont menées de manière disjointe.

28. Cette réévaluation atteint jusqu'à $23 \%$ en Haute-Volta (République de Haute-Volta, ministère de I'Économie nationale, « Compte-rendu de l'exécution de l'enquête démographique par sondage, 1960$1961 »$, Ouagadougou, 1962).

29. L'étude de 1953 n'est pas publiée. 
30. CAOM, 1fides/69, Direction des Affaires économiques et du Plan du ministère de la France d'outremer, "Essai de détermination du revenu national des principaux territoires d'outre-mer (AOF, Cameroun, AEF, Madagascar) en 1947 et en 1953 », Paris, 1955.

31. Ibid.

32. CAOM, 1affpol/2186, «L'information de la France d'outre-mer », 23-24 septembre 1956.

33. CAOM, BIB-SOM, Direction générale des Finances de l'AOF, «Essai de récapitulation des éléments connus à Dakar pour servir à un calcul du revenu national de l'AOF », Dakar, 1953.

34. ANS, 17G611, Gouvernement général de l'AOF, rapports et documentation sur les congrès interterritoriaux des partis politiques, 1956.

35. ANS, 17G272, Gouvernement général de l'AOF, rapports et documentation sur l'activité syndicale en AOF, 1955.

\section{Références}

Ageron, R., dir. 1990. Histoire de la France coloniale. 2 tomes. Paris : Armand Colin.

Alpha Gado, B. 1988. "Sécheresses et famines au Sahel. Crises alimentaires et stratégies d'autosubsistance en Afrique sahélienne. » 3 tomes. Thèse de troisième cycle, Université de Paris 7.

Ancian, G. 1952. Budgets et niveaux de vie des cultivateurs betsiléos. Paris : École nationale de la France d'Outre-Mer.

Bado, J.-P. 1996. Médecine coloniale et grandes endémies en Afrique. Paris : Karthala.

Bonnecase, V. 2009. " Avoir faim en AOF. Investigations et représentations coloniales (1920-1960). » Revue d'histoire des sciences humaines 2009 (21) : 151-174.

Bonnecase, V. 2011. La pauvreté au Sahel. Du savoir colonial à la mesure internationale. Paris : Karthala.

Bourmaud, P., dir. 2011. De la mesure à la norme : les indicateurs de développement. BangkokLausanne : Presse de l'Institut français du Proche-Orient - Bangkok Services Network.

Brian, É. 1989. "Statistique administrative et internationalisme statistique pendant la seconde moitié du XIXe siècle. » Histoire et mesures 4 (3/4) : 201-224.

Capet, M., et R. Fabre. 1957. L'économie de l'AOF depuis la guerre. Paris : Imprimerie Guillemot et de Lamothe.

Cartier, R. 1956. « En Afrique noire avec Raymond Cartier. La France sème ses milliards, les Africains disent : c'est bien tard. » Paris Match, $n^{\circ}$ 384, 25 août. 
Castro, J. de. 1951. Geopolítica da fome. Rio de Janeiro : Casa do Estudante do Brasil.

Cooper, F. 2004. Décolonisation et travail en Afrique. L'Afrique britannique et française. Paris : Karthala.

Coquery-Vidrovitch, C. 1982. "Le financement de la "mise en valeur" coloniale : méthode et premiers résultats. „ Dans Études africaines offertes à Henri Brunschwig, dirigé par J. Vansina, C.H. Perrot, VR. Austen, et Y. Person et al., 237-251. Paris : Éditions de l'École des hautes études en sciences sociales.

Data, L. 2009. Le grand trucage. Comment le gouvernement manipule les statistiques. Paris : La Découverte.

Deane, P. 1948. "The Measurement of Colonial National Incomes: An Experiment." National Institute of Economic and Social Research, Occasional Papers 1948 (7). Cambridge : Cambridge University Press.

Desrosières, A. 1994. La politique des grands nombres. Histoire de la raison statistique. Paris : La Découverte.

Desrosières, A. 2003a. "Historiciser l'action publique : I'État, le marché et les statistiques. " Dans Historicités de l'action publique, dirigé par P. Laborier et D. Trom, 207-221. Paris : Presses Universitaires de France.

Desrosières, A. 2003b. " Naissance d'un nouveau langage statistique entre 1940 et 1960. » Courrier des statistiques 2003 (198) : 41-52.

Desrosières, A. 2008a. Pour une sociologie historique de la quantification. L'argument statistique I. Paris : Presses de l'école des mines.

Desrosières, A. 2008b. Gouverner par les nombres. L'argument statistique II. Paris : Presses de l'école des mines.

Desrosières, A. 2012. « La mesure du développement. » Travail présenté à Conférence d'introduction au colloque du GEMDEV/UNESCO, Paris, 1-3 février.

Destremeau, B., et P. Salama. 2002. Mesures et démesure de la pauvreté. Paris : Presses Universitaires de France.

Fabre, R., G. Dubois, et M. Courcier. 1958. «Comptes économiques 1951-1956. Compte-rendu sommaire de travaux effectués depuis 1951, concernant certains États d'Afrique et de Madagascar. » Tiré à part d'Outre-mer, édité par le service de la Statistique du ministère de la France d'outre-mer.

Ferguson, J. 1990. The Anti-Politics Machine: "Development", Depolitization and Bureaucratic Power in Lesotho. Cambridge : Cambridge University Press. 
Fitoussi, J.-P., A. Sen, et J. E. Stiglitz, dir. 2009. Rapport de la Commission sur la mesure des performances économiques et du progrès social. Paris : Ministère français de l'Économie, de I'Industrie et de l'Emploi.

Fourquet, N., dir. 1980. Les Comptes de la puissance : histoire de la comptabilité nationale et du Plan. Paris : Éditions Recherches, coll. Encres.

Hardy, G., et C. Richet, dir. 1933. L'alimentation indigène dans les colonies françaises, protectorats et territoires sous mandats. Paris : Vigot frères éditeurs.

Haubert, M., dir. 1969. Matériaux pour une analyse de la croissance dans l'Ouest africain. Paris : Institut d'étude du développement économique et social.

Hibou, B. 2011. "Macroéconomie et domination politique en Tunisie. Du "miracle économique" benaliste aux enjeux socio-économiques du moment révolutionnaire. » Politique africaine 124 : 127154.

Horvath, R. 1972. "Le concept de statistique internationale et son évolution historique. " Revue Internationale de Statistique 40 (3) : 281-298.

Jerven, M. 2009. “The Relativity of Poverty and Income: How Reliable Are African Economic Statistics?” African Affairs 109 (434): 77-96.

Jerven, M. 2010. "Random Growth in Africa? Lessons From an Evaluation of the Growth. Evidence on Botswana, Kenya, Tanzania and Zambia, 1965-1995." Journal of Development Studies 46 (2): 274294.

Jerven, M. 2011. "Users and Producers of African Income: Measuring the Progress of African Economies." African Affairs 110 (439): 169-190.

Kent, J. 1992. The Internationalization of Colonialism. Britain, France and Black Africa, 19391956. Oxford: Clarendon Press.

Leveugle, J. 1953. Étude du revenu et des comptes du Cameroun en 1951 : principaux résultats, méthodes. Paris : Ministère de la France d'outre-mer.

Reynaud-Paligot, C. 2006. "Usages coloniaux des représentations raciales (1880-1930). Cahiers d'histoire. Revue d'histoire critique 2006 (99) : 103-110.

Rist, G. 1996. Le développement. Histoire d'une croyance occidentale. Paris : Presses de Sciences Po.

Studenski, P. 1958. The Income of Nations. Theory, Measurement, and Analysis: Past and Present. New York : New York University Press.

Vanoli, A. 2002. Une histoire de la comptabilité nationale. Paris : La Découverte. 